\title{
Germanica
}

\section{Timişoara, la petite Vienne du Banat}

Temeschwar, das kleine Wien des Banats

\section{Cécile Kovácsházy}

\section{(2) OpenEdition}

\section{Journals}

Édition électronique

URL : http://journals.openedition.org/germanica/565

DOI : 10.4000/germanica.565

ISSN : 2107-0784

Éditeur

Université de Lille

\section{Édition imprimée}

Date de publication : 1 décembre 2008

Pagination : 105-114

ISBN : 978-2-913857-22-6

ISSN : 0984-2632

Référence électronique

Cécile Kovácsházy, «Timişoara, la petite Vienne du Banat », Germanica [En ligne], 43 | 2008, mis en ligne le 01 décembre 2010, consulté le 06 octobre 2020. URL : http://journals.openedition.org/ germanica/565 ; DOI : https://doi.org/10.4000/germanica.565

Ce document a été généré automatiquement le 6 octobre 2020.

(c) Tous droits réservés 


\section{Timişoara, la petite Vienne du Banat}

Temeschwar, das kleine Wien des Banats

Cécile Kovácsházy

113 octobre 1716 : l'armée des Habsbourg, dirigée par Eugène de Savoie, prend aux mains des Turcs la ville de Temeschburg / Timişoara / Temeschwar. Le général Mercy d'Argenteau en devient le premier gouverneur ${ }^{1}$. La région du Banat, dont Timişoara est la capitale, après avoir été aux confins de l'Empire ottoman, se retrouve aux confins de l'Empire des Habsbourg, dans les Grenzregimente. L'empereur Charles VI fait alors envoyer dans cette région un grand nombre d'Allemands paysans, essentiellement issus de la Souabe, afin d'assécher les marécages et de développer une économie rurale agricole ${ }^{2}$.

28 février 1867 : un Compromis entre Vienne et Budapest est signé. On le sait, avec ce compromis, la Hongrie obtient une plus grande autonomie, donc une plus grande puissance politique. À partir des années 1880, la politique de magyarisation du royaume se durcit à l'égard des minorités ; cette magyarisation devient active voire agressive ou oppressive.

3 Juillet 2007 : j'assiste à Timişoara à un festival municipal de musique dont l'estrade est dressée sur la fameuse PiaŢa Unirii. Au-dessus de l'estrade est tendue une grande banderole, c'est le nom du festival, avec la formule suivante écrite en roumain: Timişoara: mica Viena («Timişoara: petite Vienne»). «Petite Vienne» est une expression qu'on trouve souvent accolée au toponyme de Timişoara. Je demande à Adriana, l'amie roumaine d'une cinquantaine d'années qui m'accompagne, une autochtone, universitaire, ce qu'elle pense de cette égide. Sa réponse est claire : «c'est tout à fait ridicule ».

4 Ces trois dates montrent le rapport serré existant entre Timişoara et Vienne depuis plusieurs siècles, politiquement et culturellement. Ce rapport perdure semble-t-il jusqu'aujourd'hui. 
J'étudierai ici les rapports culturels que Timişoara entretient avec Vienne autour de 1900, et plus particulièrement en ce qui concerne la population allemande du Banat. Je commencerai par rappeler en amont les raisons historiques qui expliquent que des Allemands de la capitale du Banat tissent des liens avec la capitale de l'Empire. Puis je regarderai comment la vie culturelle et intellectuelle fonctionne à Timişoara autour de 1900, dans la mesure où Vienne représente alors pour les uns un repoussoir, mais pour la majorité un modèle et un but.

6 Etrangement rarement abordée dans les livres de synthèse qui se consacrent aux littératures d'Europe centrale ${ }^{3}$, Timişoara est pourtant un centre culturel très actif, peut-être même une tentative fructueuse de décentrement de l'axe triangulaire Vienne-Berlin-Budapest. Evidemment, la chose pourrait être avancée pour d'autres grandes villes comme Trieste ou Prague ou pour certaines métropoles régionales situées également aux confins de l'Empire: Lvov, Czernowitz ou Gdańsk. Mais la particularité de Timişoara tient à sa position géographique carrefour, ainsi qu'à un multilinguisme véritable.

\section{8-1900. les Allemands du Banat et les Hongrois}

7 Depuis le début du xvIII ${ }^{\mathrm{e}}$ siècle, de nombreux Allemands sont installés dans la région du Banat (pour la raison mentionnée plus haut). « Le gouverneur Mercy, en faisant venir des colons allemands, ne voulait pas germaniser cette contrée, mais bien plutôt la peupler de paysans et d'artisans habiles, dans le but de promouvoir les progrès des Lumières. Ces colons allemands pouvaient en effet être aussi bien des Roumains ou des Slaves [...]. Le principal, c'était qu'ils eussent assimilé, pour être capables de le répandre ensuite, le goût des Allemands pour le travail sérieux et bien fait ${ }^{4}$. » Les Allemands qui se trouvent installés dans le Banat peuvent ainsi librement parler leur langue, fréquenter des écoles germanophones, etc. Ils sont principalement catholiques (les réformés constituant alors le seul groupe interdit d'immigration dans le Banat, en vue de recatholiciser l'Empire). La langue allemande est même promue et partagée de façon bien plus large que par la seule minorité allemande :

Dans ce pays sans noblesse de sang, où l'on passe vite à la promotion par le mérite, la connaissance de l'allemand permet aux Roumains, Serbes ou autres d'accéder à une carrière militaire ou administrative. De manière significative, lorsque les premières écoles allemandes ouvrirent dans le Banat de montagne - zone soit de concentration militaire soit industrielle -, bon nombre de Roumains, même d'âge adulte, s'y inscrivirent de leur propre gré pour apprendre cette langue. Dès le début $\mathrm{du} \mathrm{XIX}^{\mathrm{e}}$ siècle, dans les livres de prière des églises roumaines, les gens notaient ce qui leur semblait digne d'intérêt, en roumain et en allemand, ce qui prouve que l'allemand était connu aussi dans les villages. Pour la plupart, les élites roumaines et serbes se formaient par des études en allemand, qui devint la langue de conversation dans les salons bourgeois ${ }^{5}$.

Parmi les Allemands du Banat du xix ${ }^{e}$ siècle, on peut distinguer deux catégories : une majorité de paysans ${ }^{6}$, issus du déplacement de population à partir de 1716 , et une minorité de bourgeois citadins, bourgeoisie qui se développe après 1806, avec l'immigration d'intellectuels qui se sont opposés à la politique expansionniste de Napoléon ${ }^{7}$, puis avec l'immigration des révolutionnaires de 1848. À ce stade, il n'existe pas encore de conscience identitaire commune parmi les Allemands du Banat - la chose est bien différente avec les Saxons de Transylvanie. 
9 Mais à partir de $1861^{8}$ et plus encore à partir de 1867 avec le Compromis, les choses changent nettement. Face à la domination hongroise, les Allemands, qui sont sans organisation fédératrice, se retrouvent sans recours. C'est à ce moment-là que se développe alors une conscience identitaire et ce, d'abord chez les paysans. Peu à peu, une conscience voire une revendication se cristallise. Cet éveil des nationalités (qui n'est pas propre aux Allemands) se généralise à partir des années 1880. L'Erweckung se développe en réaction contre le nationalisme hongrois ; la main-mise hongroise suscite en effet un sentiment de menace (bien réelle) parmi les populations allemandes. Budapest impose l'usage de la langue hongroise dans l'administration, dans les écoles et ce, aux dépens des autres langues. Cette répression trouve son comble dans la fameuse Lex Apponyi de 1907.

10 La conséquence de cette magyarisation forcée est que la minorité souabe, qui n'était pourtant pas très revendicative, se tourne alors vers Vienne pour faire contre-poids à Budapest et obtenir protection face à la véhémence des Hongrois. À cela s'ajoute «le mythe du 'bon empereur' " qui date de l'époque des "confins militaires ${ }^{9}$ (dès le XVI ${ }^{e}$ siècle), une fidélité au pouvoir légal qui perdure tant que les confins militaires sont en action, soit jusqu'en 1872. Ce regard tourné vers Vienne conduit donc les Allemands du Banat, selon les mots de Dieter Kessler, à un « conflit de loyauté entre Budapest et Vienne, entre les Magyars et les Habsbourg, qui arboraient depuis la lointaine Vienne le drapeau d'une culture allemande supérieure ${ }^{10} \%$.

11 Quant à Berlin, à l'autre extrémité de l'Europe centrale, elle ne soutenait pas les Allemands du Banat étant donné qu'elle entretenait de bons rapports avec la Hongrie dans l'optique d'une solidarité antislave. Dans le cadre du dualisme administratif austro-hongrois, pour des raisons linguistiques et selon la fascination bien connue pour la ville de l'apocalypse joyeuse, les intellectuels et artistes de Timişoara se tournent donc résolument vers Vienne.

12 Le nom que les Allemands du Banat se donnent pour se désigner eux-mêmes est révélateur de cette attraction pour Vienne. Jusqu'à la Jahrhundertwende, les Allemands du Banat s'appelaient eux-mêmes des Deutschungarn, c'est-à-dire qu'ils se définissaient comme « des Hongrois qui étaient allemands, leur langue maternelle était l'allemand et leur patrie la Hongrie. Et ce, d'autant plus que la couche élevée de Timişoara (les bourgeois, les fonctionnaires et les militaires) ne s'identifiait pas aux paysans souabes des campagnes. L'assimilation allait de soi. [...] Mais avec la magyarisation forcée, ils changent leur dénomination pour se désigner désormais sous le nom de ungarländischen Deutschen, des Allemands en pays hongrois ${ }^{11}$. "

Les liens culturels, à sens unique, se resserrent donc avec Vienne, ville de force centripète.

\section{Le contexte culturel vivace de Timişoara}

14 Déjà dans les années 1850, la culture allemande connut un essor dans le Banat. Un fait culturel significatif est l'établissement à Timişoara d'une filière de la maison de presse Wiener Staatsdruckerei dès 1850. Le marché du livre est très développé alors. Selon Virgil Nemoianu, une des caractéristiques culturelles majeures du Banat est l'« ethos de l'instruction ", qu'il explique à la fois par l'influence du néoclassicisme, l'enracinement des valeurs Biedermeier et l'influence communautariste de la doctrine catholique 
thérésienne ${ }^{12}$. Parallèlement à cet ethos de l'instruction, l'essor des journaux et des magazines est remarquable, essor qui perdure jusqu'à la Deuxième Guerre mondiale. Dès 1771 avait paru le premier journal allemand, Temeswarer Nachrichten ${ }^{13}$ devenu Temesvarer Zeitung,paru de 1852 à 1949, soit pendant presque cent ans; le quotidien copie la Wiener Zeitung dans sa structure et dans sa présentation.La Neue Temesvarer Zeitung paraît, elle, de 1868 à 1912. Entre la moitié du XIX siècle et 1918, il paraît dans le Banat (mais principalement à Timişoara) environ 300 périodiques différents en allemand et 80 journaux bi- ou trilingues ${ }^{14}$ ! Plus tard, dans l'entre-deux-guerres, ce nombre décroit mais reste impressionnant : «on recense à Timişoara 29 imprimeries, 125 périodiques en roumain, 65 en allemand, 104 en hongrois, et 1 périodique en serbe. La production éditoriale du Banat est jusqu'à la fin de la Deuxième Guerre mondiale non seulement vaste, mais aussi très diversifiée ${ }^{15} »$. Claudio Magris, qui adopte un point de vue pessimiste sur la présence allemande dans le Banat à cette époque en en soulignant le déclin, avance dans Danube qu'il n'existe que 12 journaux allemands en 1902 pour 12 hongrois et 1 roumain. Il déplore ce qu'il appelle avec Adam MüllerGuttenbrunn «la dénationalisation croissante», omettant la dimension parfois contestable des propos anti-magyars de l'écrivain ${ }^{16}$. La raison de ce déclin vient de ce que «les Souabes du Banat se laissaient volontiers assimiler, donnaient des noms hongrois à leurs enfants ou magyarisaient le leur ${ }^{17}$ ».

Comment peut-on expliquer une telle production journalistique fondée sur le modèle viennois? C'est que la culture viennoise fin-de-siècle est du côté de l'immédiateté, de l'actualité, de la diffusion d'éléments plus succincts. La ville de Karl Kraus - qui abhorre pourtant le journalisme - se reflète sur Timişoara comme un modèle du fragment, du mouvement, bref, de la modernité.

\section{Les écrivains et Vienne}

Sans étonnement, on distingue dans le Banat deux directions littéraires qui se développent durant cette seconde moitié $d u x x^{e}$ siècle: d'une part la littérature régionale, littérature qualifiée par Kessler de «banatorientiert $»^{18}$, parmi lesquels on compte des auteurs peu connus internationalement: Wilhelm Brevis, Paul Moussong, Ludwig Schmidt, Conrad Jacobus Stein, Arthur Korn. D'autre part, la littérature influencée par Vienne, tournée vers Vienne, la « Wienerisch geprägte Literatur », dans l'étude de Kessler. Dans ce second groupe, on trouve des noms relativement célèbres, puisque ces auteurs ont vu leurs œuvres diffusées dans un cercle plus large: Adam Müller-Guttenbrunn, Johann Eugen Probst, Otto Alscher, Franz Xaver Kappus, Nikolaus Schmidt, Karl Grünn en font partie.

Le polygraphe Adam Müller-Guttenbrunn, assurément le plus connu et le plus reconnu de ces écrivains originaires de Timişoara, est la «figure de proue de la littérature allemande du Banat ${ }^{19}$ ». Adam Müller-Guttenbrunn (1852 Guttenbrunn, Banat roumain - 1923 Weidling, près de Vienne) a passé la plus grande partie de sa vie à Vienne, ce qui pose d'emblée la question de l'appartenance nationale : possède-t-on l'identité de ses origines ou celle de son lieu de vie ${ }^{20}$ ? C'est un enfant bâtard, le père, issu d'une assez bonne famille, n'ayant pas eu le droit d'épouser la mère, de plus basse extraction. Cela a provoqué une honte et une gêne chez Adam jamais surmontées. Müller-Guttenbrunn a donc pour objectif un ailleurs anonyme et meilleur : ce sera la ville de Vienne. Grâce à un petit travail de fonctionnaire des télégraphes, il se fait d'abord nommer à Linz. Son 
ascension sociale et littéraire commence alors de façon irrépressible. D’abord critique littéraire à Vienne, il devient dramaturge puis finalement directeur du Raimundtheater. Il atteint la célébrité au point qu'on appose une plaque sur sa maison de naissance dès 1921 (ante mortem) et qu'on nomme une rue de Temeswar d'après lui.

Après vingt années passées en Autriche, il revient dans le Banat et sa patrie lui semble étrangère. Mais suite à ce voyage, il emplit ses œuvres de héros donauschwäbisch vaillants. C'est le cas dans Glocken der Heimat (1912), dont le sous-titre est "Siedlerroman », ou encore dans Der kleine Schwab (1910), dans Der große Schwabenzug (1913) et dans son roman le plus connu, Meister Jakob und seine Kinder (1918). « Le Banat décrit par Guttenbrunn dans ses romans historiques est essentiellement rural et patriarcal, basé sur le culte du travail, de la famille et de la tradition. C'est un lieu tantôt idyllique, où les relations interethniques sont harmonieuses, tantôt bouleversé par une histoire brutale ${ }^{21} »$.

Müller-Guttenbrunn est ainsi considéré comme le Heimatdichter, « le poète du terroir, le créateur du roman allemand d'au-delà des frontières (auslanddeutscher Roman) ${ }^{22}$ ». Pendant un temps, on a même pensé que ses Heimatbücher, interdits en Hongrie,avaient permis l'éveil des Allemands du Banat, en cristallisant leurs revendications, mais ce point de vue est relativisé aujourd'hui.

20 Liée d'amitié avec Rudolf Steiner qui fréquente son salon, Marie Eugenie Delle Grazie (1864-1931) est elle aussi dramaturge et prosatrice ${ }^{23}$. C'est à l'âge de dix ans qu'elle quitte le Banat pour s'installer à Vienne. Ses romans ont été traduits en plusieurs langues et ont été couronnés par de nombreux prix. Delle Grazie parle de sa patrie d'origine (son village d'origine est Weißkirchen / Biserica Albă / Fehértemplom) notamment dans le roman Donaukind (1918), et même dans la pièce, socialiste, Schlagende Wetter, les réminiscences du monde banatais ne sont pas absentes. Delle Grazie répétait volontiers être "fière d'être née allemande parmi des Allemands ». Si elle a contribué à l'anthologie de textes d'auteurs banatais que Müller-Guttenbrunn a publiée en 1911 sous le titre Schwaben im Osten, elle a néanmoins été considérée comme ennemie d'Etat en 1939 et finalement exclue de la communauté des auteurs souabes du sud-est.

On voit chez les deux auteurs sus-mentionnés une oscillation permanente et peut-être une réconciliation littéraire entre une appartenance banataise et une appartenance viennoise, entre un monde rural, traditionnel et une frénésie citadine, moderne (et politique pour Delle Grazie).

Franz-Xaver Kappus (1883, Temeschwar - 1966, Berlin) est un officier autrichien, en Autriche, conduit par des velléités d'écriture mais qui reste avant tout connu pour la postérité comme le destinataire des Lettres à un jeune poète de Rainer Maria Rilke. Après la Première Guerre mondiale, il est rédacteur en chef à Temeschwar, puis à partir de 1925 il est lecteur pour la maison d'édition Ullstein à Berlin où il réside jusqu'à sa mort. Lui ne se sent pas du tout banatais. Mais il place souvent ses actions dans le cadre du Banat ou dans d'autres lieux souabes danubiens, afin de donner à ses écrits (par exemple dans son roman Die lebenden Vierzehn) une dimension pittoresque. Mais «Kappus opte pour un topos moderne, la ville, en divergence totale avec la littérature souabe traditionnelle. L'action des romans se passe à Timişoara ou à Budapest, dans des espaces urbains qui sont familiers à l'auteur, et porte implicitement à l'attention du public une perception non-conventionnelle du Banat: celle de sa vie citadine ${ }^{24} »$. La 
dichotomie entre une littérature régionaliste donc rurale et une littérature cosmopolite donc citadine est ici déplacée et renouvelée.

Tous ces écrivain $\mathrm{s}^{25}$ se positionnent d'abord dans la littérature allemande et dans un second temps, seulement, dans la littérature banataise ${ }^{26}$. C'est là un paradoxe :

Le paradoxe de la littérature d'expression allemande est que la majeure partie des auteurs, bien qu'immigrés de gré ou de force à Vienne, à Berlin ou ailleurs dans le monde, aient exprimé dans leurs écrits leur indéfectible loyauté envers leur région d'origine, qui a forgé leur identité. Le Banat - souvenir, source d'inspiration, ou obsession - qu'ils emportèrent avec eux à travers le monde est un sujet sur lequel ils écrivent, ce lieu étant bon ou mauvais, d'une atemporelle simplicité ou d'une complexité tourmentée, de toute façon, un lieu où il se passe quelque chose ${ }^{27}$.

C'est ce paradoxe qui invite Laura Cheie à parler d'une "littérature insulaire " signalant par là la réappropriation d'un lieu désormais d'autant plus littérarisable qu'il est géographiquement absent.

\section{Le multilinguisme à Timişoara}

Le rapport des écrivains de Timişoara à Vienne pose des questions non seulement de définition culturelle locale mais aussi (si ce n'est pas la même chose) de choix de langues pour une activité littéraire souvent polyglotte. Ce multilinguisme constitue certainement la particularité de la littérature banataise.

Le phénomène de la multiculturalité et du multilinguisme, présenté comme un truisme pour définir l'Europe centrale, se présente de façon originale dans la région du Banat (roumain) dans la mesure où ce multilinguisme n'est pas guidé par des langues dominantes que seraient alors l'allemand, l'anglais et le français. La région du Banat, pour des raisons au départ politiques et mercantiles (Empire des Habsbourg), réunit depuis le $\mathrm{XVIII}^{\mathrm{e}}$ siècle des cultures roumaines, hongroises, allemandes, serbes et slovaques. Ces cultures conjointes, auxquelles s'adjoignent différentes religions, et ces transferts interculturels donnent lieu à des phénomènes littéraires très particuliers, comme la profusion d'auteurs tri- voire quadrilingues dans leur production ${ }^{28}$. Le poète journaliste polyglotte Róbert Reiter / Franz Liebhard (1899-1989), dont la production est un peu postérieure à 1900, offre un modèle linguistiquement très mobile : né dans une famille slovaco-autrichienne, il possède deux langues maternelles, le hongrois et l'allemand. Il écrit tout d'abord en hongrois des poèmes d'avant-garde expressionniste et constructiviste, puis en allemand et en souabe. Magris dit de lui qu'il « avait appris à penser avec la mentalité de plusieurs peuples ${ }^{29}$ ». Sa mobilité est également visible sur le plan géographique et éditorial: Reiter publie autant à Timişoara qu'à Vienne, Kolozsvár (Cluj) ou Budapest. Cela s'oppose à la posture majoritaire selon laquelle «lorsqu'un écrivain choisit pour sa production littéraire ou universitaire une langue différente de sa langue maternelle, il est rare qu'il opte pour une autre langue de la région : seuls l'allemand, l'anglais ou le français entrent en ligne de compte ${ }^{30} »$.

La transmission de la culture viennoise et les passations culturelles se réalisent donc évidemment grâce à une langue commune. Deux originalités sont à relever : d'une part, que ce phénomène passe par deux couches de la population, l'immigration paysanne puis la couche intellectuelle et bourgeoise. D'autre part, dans une logique postcoloniale, que les Souabes danubiens autour de 1900 réagissent contre la culture immédiatement dominante (celle des Hongrois) au lieu de l'assimiler, en se rapprochant d'une autre 
culture, dans une certaine mesure elle aussi étrangère, et fantasmée comme culture protectrice et bénéfique. Tout en gardant les distinguo identitaires de région / nation / empire, les écrivains germanophones se tournent vers une culture considérée comme non-dominante pour proposer une forme d'hybridation. C'est à la génération suivante qu'un plurilinguisme véritablement décentré se développera, sans qu'une des langues fortes de l'Europe ne soit préférée (l'allemand en l'occurrence) aux dépens d'une autre (hongrois, roumain, slovaque).

28 À l'aune de Timişoara, on peut dire que la ville centre-européenne en 1900 est un espace qui s'étend au-delà de sa réalité : elle dessine des frontières imaginaires (ou plutôt elle les abolit) en faisant de Vienne un pendant évident de Timişoara.

\section{Et aujourd'hui ?}

Pour les écrivains allemands de Timişoara et du Banat, Vienne n'était pas un «lieu d'exil, parce que cette ville était considérée par beaucoup d'Allemands du Banat comme la capitale historique de la région et le centre spirituel de sa vie ${ }^{31}$ ». Néanmoins, malgré cette attractivité centripète durant la Jahrhundertwende, un multilinguisme réel (qui ne correspond pas au binôme langue maternelle / langue dominante) permet que se développe peu à peu une culture décentrée, décentrement qui fait que, malgré l'attraction pour Vienne autour de 1900, Timişoara possède et préserve une identité forte.

0 Pourquoi alors au xxI siècle un festival intitulé «Timişoara : Mica Viena »? Est-ce un effet d'« utopie rétrospective » (Le Rider) ? Ce qui allait de soi jadis semble être devenu une nostalgie délétère ou commerciale aujourd'hui.

31 Que reste-t-il alors de l'influence de Vienne sur Timişoara? Une architecture, un quartier Josefin, quelques théâtres non-roumanophones, quelques échanges économiques privilégiés. Comme dans le reste de l'aire géographique de l'Empire, la multiculturalité se perd. Timişoara est-elle aujourd'hui tournée vers une plus grande ville ? En fait, non. Son emplacement géopolitique : à la fois dans l'Europe du sud-est et près de Vienne, de Budapest, des Balkans, de la Croatie, de l'Italie lui permet d'être tournée de façon rayonnante, et non latérale.

Où vont les artistes aujourd'hui ? Richard Wagner écrit Die Muren von Wien (1990), mais il a immigré en 1987 à Berlin, comme Herta Müller, le plus fameux écrivain du début du $\mathrm{xxI}^{\mathrm{e}}$ siècle d'origine banataise. Pareillement, Johann Lippet, poète roumain contemporain auteur d'un Banater Alphabet, bien qu'il soit né en Autriche, vit actuellement en Allemagne où il a lui aussi émigré en 1987. Culturellement, Vienne n'est plus que le catalyseur d'une nostalgie fin-de-siècle, un catalyseur qui n'est plus depuis longtemps créateur d'images littéraires. 


\section{NOTES}

1. Claude Florimond Mercy d'Argenteau sera le premier gouverneur militaire du Banat de 1716 à 1734.

2. Voir Valeriu Leu : «Le 'Banat impérial' » in : A. Babeţi, D. Bechtel, C. Kovacshazy, X. Galmiche (éd.) : Le Banat: un eldorado aux confins, Paris, Cultures d'Europe centrale, hors-série $n^{\circ} 4,2007$, p. 39-50.

3. L'ouvrage de Jacques Le Rider et Fridrun Rinner (dir.), Les Littératures de langue allemande en Europe centrale des Lumières à nos jours, PUF, 1988 n'en parle pas.

4. Claudio Magris : [Danubio, 1986] Danube, trad. Jean et Marie-Noëlle Pastureau, Paris, Gallimard, 1988, p. 407.

5. Valeriu Leu : art. cit., p. 49.

6. Celle qui perdure encore de façon minoritaire aujourd'hui et que la romancière Herta Müller a décrit dans Der Mensch ist ein großer Fasan auf der Welt (1986) et dans Barfüßiger Februar (1987).

7. Voir Roxana Nubert et Ileana Pintilie-Teleaga : Mitteleuropäische Paradigmen in Südosteuropa : ein Beitrag zur Kultur der Deutschen im Banat, Wien, Praesens Verlag, 2006, p. 174-175.

8. Date de la création de la Principauté de Roumanie.

9. Voir Valeriu Leu : art. cit., p. 45.

10. Dieter Kessler : Die deutschen Literaturen Siebenbürgens, des Banates und des Buchenlandes : von der Revolution bis zum Ende des ersten Weltkrieges (1848-1918), Köln, Böhlau Verlag, 1997, p. 363.

11. Dieter Kessler : ibid., p. 362.

12. Virgil Nemoianu : «Cazul ethosului central-european » [« Le cas de l'ethos centre-européen »], in Adriana Babeţi et Cornel Ungureanu (dir.), Europa Centrală. Nevroze, dileme, utopii [L'Europe Centrale. Névroses, dilemmes, utopies], Iaşi, Polirom, 1997, p. 168-194 ; étude publiée initialement sous le titre «Learning over Classes : The Case of Central-European Ethos », in Utrecht Publications in General and Comparative Literature Series, Amsterdam/Philadelphia, John Benjamins Publishing Co., 1993, vol. 31.

13. Voir Laura Cheie : "Insularité des écrivains germanophones du Banat?", in : Le Banat : un Eldorado aux confins, op. cit., p. 130.

14. Dieter Kessler : op. cit., p. 377.

15. Adriana BabeŢi : «Le Banat: un paradis aux confins ", in : Le Banat : un Eldorado aux confins, op. cit., p. 25.

16. Concernant la double réception de l'auteur, voir la synthèse faite par Laura Cheie, art. cit., p. 133-134.

17. Claudio Magris : op. cit., p. 424.

18. Dieter Kessler, op. cit.

19. Laura Cheie, art. cit., p. 133.

20. Dans la sphère centre-européenne, nombreux sont les auteurs dont l'identité nationale est ainsi difficilement assignable, que l'on songe, parmi tant d'autres, à Elias Canetti ou à Agota Kristof

21. Laura Cheie : art. cit., p. 133.

22. Anton Scherer: Geschichte der donauschwäbischen Literatur von 1848 bis 2000, Graz, Donauschwäbisches Bibliographisches Archiv, 2003, p. 17.

23. On accède à une bio-bibliographie sur Eugenie Delle Grazie sur le site http://www2.onb.ac.at/ ariadne/vfb/bio_dellegrazie.htm

24. Laura Cheie : art. cit., 135. 
25. On pourrait encore citer Stephan Milow, l'auteur des Fallende Blätter, poésie que Rilke, son ami, admire. Sur Milow, voir Roxana Nubert et Ileana Pintilie-Teleaga: Mitteleuropäische Paradigmen in Südosteuropa : ein Beitrag zur Kultur der Deutschen im Banat, op. cit., p. 199-207).

Nikolaus Lenau n'est en revanche pas à considérer comme un auteur banatais puisqu'il a quitté cette région avant l'âge d'un an.

26. Voir Dieter Kessler : op. cit., p. 422.

27. Laura Cheie : art. cit., p. 131.

28. Andreas Lillin, par exemple, écrit indifféremment en serbe, allemand, hongrois et roumain.

29. Claudio Magris : op. cit., p. 402.

On trouve quelques poèmes de Róbert Reiter en français dans Le Banat: un Eldorado aux confins, op. cit.

30. Jacques Le Rider et Fridrun Rinner : op. cit., p. 31.

31. Laura Cheie : art. cit., p. 133.

\section{RÉSUMÉS}

Au début du XVIII siècle, le Banat a vu l'installation de Souabes dans ces confins. Autour de 1900, ces Allemands du Banat, et particulièrement les écrivains, entretiennent un lien privilégié avec Vienne. Cette focalisation est notamment due à la magyarisation forcée (après le Compromis de 1867), qui pousse la population allemande du Banat à se définir ethniquement comme minorité. Des écrivains comme Adam Müller-Guttenbrunn, Franz-Xaver Kappus ou Eugenie Delle Grazie partent faire carrière à Vienne, et se font à la fois les chantres d'une littérature régionaliste, banataise, et d'une littérature plus viennoise et cosmopolite. Cette esthétique bicéphale s'explique notamment par la forte tradition multiculturelle de la population banataise et la vie intellectuelle et journalistique effervescente de Timişoara à cette époque.

Am Anfang des 18. Jahrhunderts wanderten Deutsche in die Grenzregionen des Kaisertums ein in den Banat. Um die Jahrhundertwende pflegen diese ,Donauschwaben', besonders SchriftstellerInnen, eine privilegierte Verbindung mit Wien. Diese Konzentration auf Wien hat ihren Grund vor allem in der Magyarisierung innerhalb der ungarischen Gebiete der Habsburgermonarchie (nach dem Ausgleich von 1867), durch die die deutsche Bevölkerung des Banats sich gezwungen fühlt, sich als ethnische Minderheit zu definieren. SchriftstellerInnen wie Adam Müller-Guttenbrunn, Franz-Xaver Kappus oder Eugenie Delle Grazie gehen nach Wien und machen dort Karriere, wobei sie in Wien gleichzeitig als 'Vertreter' einer regionalistischen, banatischen Literatur und auch einer wienerischen, kosmopolitischen Literatur wahrgenommen werden. Diese zweifache Ästhetik erklärt sich insbesondere durch die starke multikulturelle Tradition der banatischen Bevölkerung und durch das prickelnde intellektuelle und journalistische Leben in Timişoara in dieser Zeit.

\section{INDEX}

Mots-clés : Timişoara 
AUTEUR

CÉCILE KOVÁCSHÁZY

Université de Limoges et Freie Universität Berlin 